\title{
The study of the gluon distribution function and reduced cross section behavior using the proton structure function
}

\author{
B.Rezae** and G.R.Boroun \\ Physics Department, Razi University, Kermanshah 67149, Iran
}

(Dated: June 30, 2020)

\begin{abstract}
The behavior of the gluon distribution function and the reduced cross section considered from the proton structure function and its derivatives at low values of $x$. These behaviors studied and compared with the experimental data. These results are augmented by including an additional higher-twist term in the description of the nonlinear correction. This additional term, modified nonlinear correction, improves the description of the reduced cross sections significantly at low values of $Q^{2}$. We discuss, furthermore, how this behavior can be determine the reduced cross section with respect to the proton parameterization at high-y values. The resulting predictions for $\sigma_{r}$ suggest that further corrections are required for $Q^{2}$ less than about $3 \mathrm{GeV}^{2}$.
\end{abstract}

\section{Introduction}

The gluon distribution in hadrons at small Bjorken $x$ play a vital role in our understanding of the deepinelastic scattering (DIS) and our predictions for new physics at the Large Hadron Collider (LHC) in ultrahigh energy processes. It is important to know the gluon distribution inside a hadron at small $x$ because gluons are expected to be dominant in this region. The gluons couple only through the strong interaction. Consequently the gluons cannot be measured directly in the DIS. Indeed, the gluon density is much higher than of charged partons and the photoabsorbtion will be dominated by the boson-gluon fusion (BGF) $\gamma^{*} p \rightarrow q \bar{q}$ [1].

The high-luminosity LHC program would be uniquely complemented by the proposed Large Hadron electron Collider ( $\mathrm{LHeC}$ ), where it is a high-energy lepton-proton and lepton-nucleus collider based at CERN [2]. The kinematic range in the $\left(x, Q^{2}\right)$ plane of the $\mathrm{LHeC}$ for electron and positron neutral-current $(\mathrm{NC})$ for the high energy $E_{p}=7 \mathrm{TeV}$ is $5 \times 10^{-6} \leq x \leq 0.8$ and $5 \leq Q^{2} \leq 10^{6} \mathrm{GeV}^{2}$ and $5 \leq Q^{2} \leq 5 \times 10^{5} \mathrm{GeV}^{2}$ respectively. Today, an integrated Future Circular Collider programme consisting of a luminosity-frontier highest-energy lepton collider followed by an energyfrontier hadron collider is called FCC [3]. In this collider the FCC-eh with $50 \mathrm{TeV}$ proton beams colliding with $60 \mathrm{GeV}$ electrons from an energy-recovery linac would generate $\sim 2 \mathrm{ab}^{-1}$ of $3.5 \mathrm{TeV}$ ep collisions. Deep inelastic scattering measurements at FCC-eh will allow the determination of the gluon saturation phenomena required to unitarise the high-energy cross sections. The determination of the high gluon densities and non-linear

\footnotetext{
*brezaei@razi.ac.ir

†Electronic address: grboroun@gmail.com; boroun@razi.ac.ir
}

dynamics at very small $x$ is relevant to the interactions of cosmic ultra high energy neutrinos. For HERA data, the gluon density is not determined at low $x$. But, with the FCC-eh, a precision of a few percent at small $x$ becomes possible down to nearly $x \simeq 10^{-6}$ [3].

The gluon and quark distributions are determined mainly by the proton structure function $F_{2}^{\gamma p}\left(x, Q^{2}\right)$ measured in ep DIS. The longitudinal structure function and the slope of the transverse structure function become direct probes of the gluon and sea quark distributions, over a large domain of values of $x$ and $Q^{2}$ (hereafter $Q^{2}$ is the virtuality of the photon). Due to its origin, $F_{L}$ is directly sensitive to the gluon distribution in the proton and consequently it is an important quantity [4]. In perturbative quantum chromodynamics (pQCD) the heavy quark production at HERA proceeds is dominantly via the direct BGF. Therefore, once the distribution of the gluon inside the proton is known, the heavy quark distribution can be easy calculated from it. This process can be created when the squared invariant mass of the hadronic final state has the condition the runs as follows $W^{2} \geq 4 m_{\text {Heavy-quark. At }}^{2}$ very low $x$, the parton density cannot grow forever because hadronic cross-sections comply with the unitary bound known as Froissart Bound [5]. For this region the gluon recombination is known to provide the mechanism responsible for the unitarization of the cross section at high energies. In other words, the multiple gluon-gluon interactions provide nonlinear corrections in the DGLAP evolution equations [6]. While the precise measurement of $F_{L}$ at the FCC-eh and $F_{2}$ can discover whether gluon saturates.

We know that the DGLAP evolution equations are the basic perturbative tools to study the $Q^{2}$ evolution of parton distribution functions (PDFs) in the deep inelastic regime. The solutions of the coupled DGLAP evolution equations will provide the gluon and singlet quark distributions inside the nucleon at small $x$. These distribution functions have been 
determined simultaneously by starting with a virtuality $Q_{0}^{2} \leq\left(m_{c}^{2} \approx 2 \mathrm{GeV}^{2}\right)$ [7]. The initial distributions for the parton distributions usually are determined in a global QCD analysis including a wide range of DIS data from collider until fixed experiments. In Ref.[8], a new form of the DIS structure function (SF) $F_{2}\left(x, Q^{2}\right)$ in the domain $10^{-3} \leq x \leq 0.09$ and $0.11 \mathrm{GeV}^{2} \leq Q^{2} \leq 1200 \mathrm{GeV}^{2}$ of HERA data was proposed. This new form of the proton structure function leads to the small $x$ asymptotics of the reduced cross-sections $\sim \ln ^{2} 1 / x$, which is in turn in an agreement with the Froissard predictions [5]. The modified form of the proton structure function with a new parametrization which describes fairly well the available experimental data, at asymptotically small $x$, have been presented in Refs. [9-10]. This parametrization suggested is pertinent in investigations of lepton-hadron processes at ultra-high energies, which obtained from a combined fit of the H1 and ZEUS collaborations data [11] in a range of the kinematical variables $x$ and $Q^{2}$, $x \leq 0.1$ and $0.15 \mathrm{GeV}^{2} \leq Q^{2} \leq 3000 \mathrm{GeV}^{2}$.

In Ref.[12], an analytical relation has been derived for calculating the gluon distribution function within the Laplace-transform method at leading order. The gluon distribution is determined by the parametrization of $F_{2}$ (Ref.[8]) as a simultaneous function of $x$ and $Q^{2}$ in a domain $x_{\min } \leq x \leq x_{\max }$ and $Q_{\min }^{2} \leq Q^{2} \leq Q_{\max }^{2}$. Then, in Ref.[13], authors extended those previous derivation of the gluon distribution at leading order to include the effects of heavy-quark mass. These analytical solutions of the gluon behavior using a differntial-equation method [12] and a Laplace transform technique [13] have been reported with considerable phenomenological success at leading order (LO) approximation respectively. Also the solutions of the unpolarized DGLAP equation for the QCD evolution of gluon distribution have been discussed considerably over the past years $[14,15]$. In two recent papers [16-17] the behavior of the gluon distribution function $G\left(x, Q^{2}\right)$ studied at small values of $x$ by using a global parameterization of the data on $F_{2}\left(x, Q^{2}\right)$ at LO analysis.

In the present paper we study the behavior of the gluon distribution function using the proton structure function and its derivatives with respect to $\ln Q^{2}$ in high-order corrections based on the hard-pomeron exchange [4,18]. Then we estimate the nonlinear corrections to the gluon distribution function behavior at small values of $x$. Therefore, to obtain a precise evidence of the nonlinear corrections in the HERA kinematic region, we consider the longitudinal structure function that directly depends on the behavior of the gluon distribution in which the active quarks are treated as massless. Then we generalize to the case of reduced cross section using the same method. We use the modified nonlinear corrections and higher twist predictions in obtaining our analytical solutions at low $Q^{2}$ and low $x$. A completed comparison between the obtained results at NNLO approximation and available DIS data are presented.

\section{Analytical treatment of the gluon distribution function}

The DGLAP evolution equation for the singlet quark structure function is given by

$$
\begin{aligned}
\frac{\partial F_{2}^{s}\left(x, Q^{2}\right)}{\partial \ln Q^{2}}= & \frac{\alpha_{s}}{2 \pi} \int_{x}^{1} d z\left[P_{q q}\left(z, \alpha_{s}\left(Q^{2}\right)\right) F_{2}^{s}\left(\frac{x}{z}, Q^{2}\right)\right. \\
& \left.+2 N_{f} P_{q g}\left(z, \alpha_{s}\left(Q^{2}\right)\right) G\left(\frac{x}{z}, Q^{2}\right)\right]
\end{aligned}
$$

where $F_{2}^{s}\left(x, Q^{2}\right)$ and $G\left(x, Q^{2}\right)$ are singlet and gluon distribution functions. Here the representation for the gluon distribution $G\left(x, Q^{2}\right)=x g\left(x, Q^{2}\right)$ is used where $g\left(x, Q^{2}\right)$ is the gluon density. The splitting functions $P_{i j} s$ are the LO, NLO and NNLO Altarelli- Parisi splitting kernels as

$$
\begin{aligned}
P_{i j}\left(x, \alpha_{s}\left(Q^{2}\right)\right)= & P_{i j}^{\mathrm{LO}}(x)+\frac{\alpha_{s}\left(Q^{2}\right)}{2 \pi} P_{i j}^{\mathrm{NLO}}(x) \\
& +\left(\frac{\alpha_{s}\left(Q^{2}\right)}{2 \pi}\right)^{2} P_{i j}^{\mathrm{NNLO}}(x),
\end{aligned}
$$

which the explicit forms of the splitting functions at LO upto NNLO are given in Refs.[19-20].

The running coupling constant $\alpha_{s}$ has the following forms in LO, NLO and NNLO approximation respectively [21]

$$
\begin{gathered}
\alpha_{s}^{\mathrm{LO}}=\frac{4 \pi}{\beta_{0} t}, \\
\alpha_{s}^{\mathrm{NLO}}=\frac{4 \pi}{\beta_{0} t}\left[1-\frac{\beta_{1} \ln t}{\beta_{0}^{2} t}\right],
\end{gathered}
$$

and

$$
\begin{aligned}
\alpha_{s}^{\mathrm{NNLO}}= & \frac{4 \pi}{\beta_{0} t}\left[1-\frac{\beta_{1} \ln t}{\beta_{0}^{2} t}+\frac{1}{\left(\beta_{0} t\right)^{2}}\left[\left(\frac{\beta_{1}}{\beta_{0}}\right)^{2}\right.\right. \\
& \left.\left.\left(\ln ^{2} t-\ln t+1\right)+\frac{\beta_{2}}{\beta_{0}}\right]\right] .
\end{aligned}
$$

where $\beta_{0}=\frac{1}{3}\left(33-2 n_{f}\right), \beta_{1}=102-\frac{38}{3} n_{f}$ and $\beta_{2}=$ $\frac{2857}{6}-\frac{6673}{18} n_{f}+\frac{325}{54} n_{f}^{2}$. The variable $t$ is defined as $t=\ln \left(\frac{Q^{2}}{\Lambda^{2}}\right)$ and $\Lambda$ is the QCD cut- off parameter at each heavy quark mass threshold as we take the $n_{f}=4$ for $m_{c}^{2}<\mu^{2}<m_{b}^{2}$.

The power law behavior of the singlet and gluon distribution functions introduced as $F_{2}^{s} \sim x^{-\lambda_{s}}$ and $G \sim x^{-\lambda_{g}}[4,18]$ where exponents $\lambda_{s}$ and $\lambda_{g}$ are given as the derivatives: $\lambda_{s}=\frac{\partial \ln F_{2}^{s}\left(x, Q^{2}\right)}{\partial \ln (1 / x)}$ and $\lambda_{g}=\frac{\partial \ln G\left(x, Q^{2}\right)}{\partial \ln (1 / x)}$. By considering the hard-power behavior of the singlet and gluon distribution functions, one can rewrite the evolution equation 
in terms the convolution integrals, using the convolution symbol $\otimes$, as

$$
\begin{aligned}
\frac{\partial F_{2}\left(x, Q^{2}\right)}{\partial \ln Q^{2}}= & F_{2}\left(x, Q^{2}\right) \Phi_{q q}\left(x, Q^{2}\right) \\
& +G\left(x, Q^{2}\right) \Theta_{q g}\left(x, Q^{2}\right),
\end{aligned}
$$

where $F_{2}=\frac{5}{18} F_{2}^{s}$ and the nonsinglet contribution is negligible at small $x$ and can be ignored. The kernels for the quark and gluon sectors, denoted by $\Phi$ and $\Theta$, presented at LO up to NNLO respectively as

$$
\begin{aligned}
& \Theta_{q g}\left(x, Q^{2}\right)=P_{q g}\left(x, \alpha_{s}\right) \odot x^{\lambda_{g}}, \\
& \Phi_{q q}\left(x, Q^{2}\right)=P_{q q}\left(x, \alpha_{s}\right) \odot x^{\lambda_{s}},
\end{aligned}
$$

where $f(x) \odot g(x) \equiv \int_{x}^{1}(d y / y) f(y) g(y)$. The singlet exponent $\lambda_{s}$ is found to be $\simeq 0.33$ in Refs. [22-23] and the gluon exponent $\lambda_{g}$ is found to be $\simeq 0.43[4,18,23]$. Therefore the gluon distribution function is defined by the following form

$$
G\left(x, Q^{2}\right)=\frac{1}{\Theta_{q g}\left(x, Q^{2}\right)}\left[\frac{\partial F_{2}\left(x, Q^{2}\right)}{\partial \ln Q^{2}}-\Phi_{q q}\left(x, Q^{2}\right) F_{2}\left(x, Q^{2}\right)\right],
$$

where the parameterization $F_{2}\left(x, Q^{2}\right)$ and its derivatives suggested in Refs. [8-10]. This result is general and simple and gives the leading order upto high-order expressions for the gluon distribution once the parameterization $F_{2}$ is known. This relation primarily comes from the extension of range and precision in the measurements of $F_{2}\left(x, Q^{2}\right)$ and $\frac{\partial F_{2}\left(x, Q^{2}\right)}{\partial \ln Q^{2}}$, which at small $x$ are measures of the gluon density.

We write our final analytical answer by the following form as

$$
G\left(x, Q^{2}\right)=\frac{1}{\left\{P_{q g}\left(x, \alpha_{s}\right) \odot x^{\lambda_{g}}\right\}}\left[\frac{\partial F_{2}\left(x, Q^{2}\right)}{\partial \ln Q^{2}}-\left\{P_{q q}\left(x, \alpha_{s}\right) \odot x^{\lambda_{s}}\right\} F_{2}\left(x, Q^{2}\right)\right] .
$$

The gluon distribution function at leading-order analysis is given by

$$
\begin{aligned}
G^{\mathrm{LO}}\left(x, Q^{2}\right)= & \frac{\frac{9 \pi}{5 \alpha_{s}}}{\int_{x}^{1}\left(z^{2}+(1-z)^{2}\right) z^{\lambda_{g}} d z}\left[\frac{\partial F_{2}\left(x, Q^{2}\right)}{\partial \ln Q^{2}}-\left(\frac { \alpha _ { s } } { 4 \pi } \left\{4+\frac{16}{3} \ln \left(\frac{1-x}{x}\right)+\frac{16}{3} \int_{x}^{1} \frac{z^{\lambda_{s}}-z^{-1}}{1-z} d z\right.\right.\right. \\
& \left.\left.\left.-\frac{8}{3} \int_{x}^{1}(1+z) z^{\lambda_{s}} d z\right\}\right) F_{2}\left(x, Q^{2}\right)\right] .
\end{aligned}
$$

The relations in Eqs.(8) until (10) give the leading order and high-order expression for gluon distribution function in terms of a function determined by the proton structure function.

\section{Further consideration}

We now discuss how the presented results give the independent evolution equation for the singlet structure function at small $x$. The DGLAP evolution equation for the gluon distribution is given by

$$
\begin{aligned}
\frac{\partial G\left(x, Q^{2}\right)}{\partial \ln Q^{2}}= & G\left(x, Q^{2}\right) \Phi_{g g}\left(x, Q^{2}\right) \\
& +F_{2}\left(x, Q^{2}\right) \Theta_{g q}\left(x, Q^{2}\right),
\end{aligned}
$$

where kernels are

$$
\begin{aligned}
& \Theta_{g q}\left(x, Q^{2}\right)=P_{g q}\left(x, \alpha_{s}\right) \odot x^{\lambda_{s}}, \\
& \Phi_{g g}\left(x, Q^{2}\right)=P_{g g}\left(x, \alpha_{s}\right) \odot x^{\lambda_{g}} .
\end{aligned}
$$

Let us consider the evolution of the gluon distribution $G\left(x, Q^{2}\right)$ (Eq.8) with respect to $\ln Q^{2}$ :

\section{For fixed coupling case}

$$
\begin{aligned}
\frac{\partial G\left(x, Q^{2}\right)}{\partial \ln Q^{2}}= & \frac{1}{\Theta_{q g}\left(x, Q^{2}\right)}\left[\frac{\partial^{2} F_{2}\left(x, Q^{2}\right)}{\partial \ln ^{2} Q^{2}}\right. \\
& \left.-\Phi_{q q}\left(x, Q^{2}\right) \frac{\partial F_{2}\left(x, Q^{2}\right)}{\partial \ln Q^{2}}\right] .
\end{aligned}
$$


After some rearranging a homogeneous second-order differential equation is found. It is determined the proton structure function $F_{2}\left(x, Q^{2}\right)$ without having knowledge in terms of the gluon distribution function, as

$$
\frac{\partial^{2} F_{2}\left(x, Q^{2}\right)}{\partial \ln ^{2} Q^{2}}-\left[\Phi_{q q}\left(x, Q^{2}\right)+\Phi_{g g}\left(x, Q^{2}\right)\right] \frac{\partial F_{2}\left(x, Q^{2}\right)}{\partial \ln Q^{2}}+\left[\Phi_{q q}\left(x, Q^{2}\right) \Phi_{g g}\left(x, Q^{2}\right)-\Theta_{q g}\left(x, Q^{2}\right) \Theta_{g q}\left(x, Q^{2}\right)\right] F_{2}\left(x, Q^{2}\right)=0
$$

For running coupling case
The evolution of the gluon distribution $G\left(x, Q^{2}\right)($ Eq.8) with respect to $\ln Q^{2}$ is given by

$$
\begin{aligned}
\frac{\partial G\left(x, Q^{2}\right)}{\partial \ln Q^{2}}= & \frac{\partial}{\partial \ln Q^{2}}\left(\frac{1}{\Theta_{q g}\left(x, Q^{2}\right)}\right)\left[\frac{\partial F_{2}\left(x, Q^{2}\right)}{\partial \ln Q^{2}}-\Phi_{q q}\left(x, Q^{2}\right) F_{2}\left(x, Q^{2}\right)\right]+\frac{1}{\Theta_{q g}\left(x, Q^{2}\right)}\left[\frac{\partial^{2} F_{2}\left(x, Q^{2}\right)}{\partial \ln ^{2} Q^{2}}\right. \\
& \left.-\frac{\partial \Phi_{q q}\left(x, Q^{2}\right)}{\partial \ln Q^{2}} F_{2}\left(x, Q^{2}\right)-\Phi_{q q}\left(x, Q^{2}\right) \frac{\partial F_{2}\left(x, Q^{2}\right)}{\partial \ln Q^{2}}\right] .
\end{aligned}
$$

In this case the evolution equation for the singlet struc-

ture function decoupled takes the form

$$
\begin{aligned}
& \frac{\partial^{2} F_{2}\left(x, Q^{2}\right)}{\partial \ln ^{2} Q^{2}}-\left[\frac{\partial \ln \Theta_{q g}\left(x, Q^{2}\right)}{\partial \ln Q^{2}}+\Phi_{q q}\left(x, Q^{2}\right)+\Phi_{g g}\left(x, Q^{2}\right)\right] \frac{\partial F_{2}\left(x, Q^{2}\right)}{\partial \ln Q^{2}} \\
& +\left[\Phi_{q q}\left(x, Q^{2}\right) \frac{\partial \ln \Theta_{q g}\left(x, Q^{2}\right)}{\partial \ln Q^{2}}-\frac{\partial \Phi_{q q}\left(x, Q^{2}\right)}{\partial \ln Q^{2}}+\Phi_{q q}\left(x, Q^{2}\right) \Phi_{g g}\left(x, Q^{2}\right)-\Theta_{q g}\left(x, Q^{2}\right) \Theta_{g q}\left(x, Q^{2}\right)\right] F_{2}\left(x, Q^{2}\right)=0
\end{aligned}
$$

\section{Solution for $\sigma_{r}\left(x, Q^{2}\right)$ using a Froissart bounded structure function $F_{2}\left(x, Q^{2}\right)$}

The reduced neutral current (NC) deep inelastic ep scattering cross sections are given by a linear combination of generalised structure functions. The proton structure functions $F_{2}$ and $F_{L}$ are related to the $\gamma^{*} p$ cross sections of longitudinally and transversely polarized photons $\sigma_{L}$ and $\sigma_{T}$ as $F_{L} \propto \sigma_{L}$ and $F_{T} \propto \sigma_{L}+\sigma_{T}$. In one photon exchange approximation, at low and moderate $Q^{2}$ (i.e., $Q^{2} \leq M_{z}^{2} \approx 800 \mathrm{GeV}^{2}$ ), the reduced cross section is defined into the transverse and longitudinal structure functions, $F_{2}\left(x, Q^{2}\right)$ and $F_{L}\left(x, Q^{2}\right)$, by the following form

$$
\sigma_{r}\left(x, Q^{2}\right)=F_{2}\left(x, Q^{2}\right)-\frac{y^{2}}{Y_{+}} F_{L}\left(x, Q^{2}\right) .
$$

Here $Y_{+}=1+(1-y)^{2}, y=Q^{2} / x s$ is the inelasticity and $s$ is the center-of-mass squared energy of incoming electrons and protons. The contribution of the term containing the longitudinal structure function $F_{L}$ is only significant for values of $y$ larger than approximately 0.5 . Indeed, the longitudinal structure function is predominant in the cross section in case of scattering of cosmic neutrinos from hadrons [24,14]. Thus, the longitudinal structure function at low $x$ will be checked in high energy process such as the Large Hadron electron Collider (LHeC) project which runs to beyond a $\mathrm{TeV}$ in centerof-mass energy [1].

In perturbative QCD, the longitudinal structure function in terms of the coefficient functions is given by [25]

$$
x^{-1} F_{L}=C_{L, n s} \otimes q_{n s}+<e^{2}>\left(C_{L, q} \otimes q_{s}+C_{L, g} \otimes g\right),
$$

where the non-singlet quark distribution, $x q_{n s}$, become negligibly small in comparison with the singlet and gluon distribution functions, $x q_{n}$ and $x g$, at low values of $x$ and can be ignored. $\left\langle e^{k}\right\rangle$ is the average of the charge $e^{k}$ for the active quark flavors, $\left.<e^{k}\right\rangle=n_{f}^{-1} \sum_{i=1}^{n_{f}} e_{i}^{k}$. The average squared charge for even $n_{f}$ is equal to $5 / 18$. The 
symbol $\otimes$ denotes a convolution according to the usual prescription, $f(x) \otimes g(x)=\int_{x}^{1} \frac{d y}{y} f(y) g\left(\frac{x}{y}\right)$. The perturbative expansion of the coefficient functions can be written as [26]

$$
C_{L, a}\left(\alpha_{s}, x\right)=\sum_{n=1} a(t)^{n} c_{L, a}^{n}(x),
$$

where $n$ is the order in the running coupling constant. The running coupling constant in the high-loop corrections of the above equation is expressed entirely thorough the variable $a(t)$, as $a(t)=\frac{\alpha_{s}}{4 \pi}$. The explicit expression for the coefficient functions in LO up to NNLO are rele- gated in Ref.[26].

Exploiting the small- $x$ behavior of the distribution functions according to the hard (Lipatov) Pomeron, then Eq.(18) can be written as

$F_{L}\left(x, Q^{2}\right)=F_{2}\left(x, Q^{2}\right) I_{L, s}\left(x, Q^{2}\right)+G\left(x, Q^{2}\right) I_{L, g}\left(x, Q^{2}\right)$

where $I_{L, s}\left(x, Q^{2}\right)=C_{L, q}\left(\alpha_{s}, x\right) \odot x^{\lambda_{s}} \quad$ and $I_{L, g}\left(x, Q^{2}\right)=<e^{2}>C_{L, g}\left(\alpha_{s}, x\right) \odot x^{\lambda_{g}}$. On this basis the reduced cross section obtain by employing the parametrization of $F_{2}\left(x, Q^{2}\right)$ as

$$
\begin{aligned}
\sigma_{r}\left(x, Q^{2}\right)= & F_{2}\left(x, Q^{2}\right)\left\{1-\frac{y^{2}}{Y_{+}} I_{L, s}\left(x, Q^{2}\right)\right\}-\frac{y^{2}}{Y_{+}} I_{L, g}\left(x, Q^{2}\right) G\left(x, Q^{2}\right)[\equiv E q .8] \\
& =F_{2}\left(x, Q^{2}\right)\left\{1-\frac{y^{2}}{Y_{+}}\left(I_{L, s}\left(x, Q^{2}\right)-\frac{\Phi_{q q}\left(x, Q^{2}\right)}{\Theta_{q g}\left(x, Q^{2}\right)} I_{L, g}\left(x, Q^{2}\right)\right)\right\}-\frac{\partial F_{2}\left(x, Q^{2}\right)}{\partial \ln Q^{2}}\left\{\frac{y^{2}}{Y_{+}} \frac{I_{L, g}\left(x, Q^{2}\right)}{\Theta_{q g}\left(x, Q^{2}\right)}\right\}
\end{aligned}
$$

Therefore an explicit equation for reduced cross section in terms of the proton structure function parametrization and its derivatives is obtained.

\section{Nonlinear and Higher Twist corrections}

\section{Nonlinear corrections:}

The screening effects are provided by a multiple gluon interaction which leads to the nonlinear terms in the derivation of the linear DGLAP evolution equations. Therefore the standard linear DGLAP evolution equations will have to be modified in order to take the nonlinear corrections into account. Indeed the origin of the shadowing correction, in pQCD interactions, is primarily considered as the gluon recombination $(g+g \rightarrow g)$ which is simply the inverse process of gluon splitting $(g \rightarrow g+g)$. Gribov, Levin, Ryskin, Mueller and Qiu (GLR-MQ) [27] performed a detailed study of these recombination processes. This widely known as the GLR-MQ equation and involves the two-gluon distribution per unit area of the hadron. This equation predicts a saturation behavior of the gluon distribution at very small $x$ [28]. A closer examination of the small $x$ scattering is resummation powers of $\alpha_{s} \ln (1 / x)$ where leads to the $k_{T}$-factorization form [29]. In the $k_{T}$-factorization approach the large logarithms $\ln (1 / x)$ are relevant for the unintegrated gluon density in a nonlinear equation. Solution of this equation develops a saturation scale where tame the gluon density behavior at low values of $x$ and this is an intrin- sic characteristic of a dense gluon system.

Therefore one should consider the low- $x$ behavior of the singlet distribution using the nonlinear GLR-MQ evolution equation. The shadowing correction to the evolution of the singlet quark distribution can be written as [30]

$$
\begin{aligned}
\frac{\partial x q\left(x, Q^{2}\right)}{\partial \ln Q^{2}}= & \left.\frac{\partial x q\left(x, Q^{2}\right)}{\partial \ln Q^{2}}\right|_{D G L A P} \\
& -\frac{27 \alpha_{s}^{2}}{160 R^{2} Q^{2}}\left[x g\left(x, Q^{2}\right)\right]^{2}
\end{aligned}
$$

Eq. (22) can be rewrite in a convenient form as

$$
\begin{aligned}
\frac{\partial F_{2}\left(x, Q^{2}\right)}{\partial \ln Q^{2}}=\left.\frac{\partial F_{2}\left(x, Q^{2}\right)}{\partial \ln Q^{2}}\right|_{D G L A P} & -\frac{5}{18} \frac{27 \alpha_{s}^{2}}{160 R^{2} Q^{2}} \\
& \times\left[x g\left(x, Q^{2}\right)\right]^{2}
\end{aligned}
$$

The first term is the standard DGLAP evolution equation and the value of $R$ is the correlation radius between two interacting gluons. It will be of the order of the proton radius $\left(R \simeq 5 \mathrm{GeV}^{-1}\right)$, if the gluons are distributed through the whole of proton, or much smaller $\left(R \simeq 2 \mathrm{GeV}^{-1}\right)$ if gluons are concentrated in hot- spot within the proton.

Also there is another mechanism to prevent generation of the high density gluon states, as this is well known the vacuum color screening [31]. There is a transition between the nonperturbative and perturbative domains. In the QCD vacuum, the non-perturbative fields form structures with sizes $\sim R_{c}$ which it is smaller than $\Lambda_{Q C D}$. The short propagation length for perturbative gluons is $R_{c} \sim 0.2-0.3 \mathrm{fm}$. The gluon fusion effect in non-linear regime controlled by the new dimensionless parameter 
$\sim \frac{R_{c}^{2}}{8 B}$ where $B$ is the characteristic size of the interaction region as this parameter can be defined by $\ln \left(x_{0} / x\right)$ and $r$ where $r^{2} \sim Q^{-2}$. Authors in this reference (i.e., Ref.[31]) show that the nonlinear effects leads to the logarithmically ratio as the nonlinear/linear effects are proportional to $R_{c}^{2} / 8 B\left(\ln \left(x_{0} / x\right), r^{2}\right) \ln \left(Q^{2} R_{c}^{2}\right)$.

Combining Eqs. (8) and (23), one could consider the nonlinear correction to the gluon distribution function as

$$
\begin{aligned}
G\left(x, Q^{2}\right)= & \frac{1}{\Theta_{q g}\left(x, Q^{2}\right)}\left[\frac{\partial F_{2}\left(x, Q^{2}\right)}{\partial \ln Q^{2}}-\frac{5}{18} \frac{27 \alpha_{s}^{2}}{160 R^{2} Q^{2}} \times\right. \\
& \left.G^{2}\left(x, Q^{2}\right)-\Phi_{q q}\left(x, Q^{2}\right) F_{2}\left(x, Q^{2}\right)\right],
\end{aligned}
$$

where

$$
\begin{aligned}
& G\left(x, Q^{2}\right)+\frac{1}{\Theta_{q g}\left(x, Q^{2}\right)} \frac{5}{18} \frac{27 \alpha_{s}^{2}}{160 R^{2} Q^{2}} G^{2}\left(x, Q^{2}\right)= \\
& \frac{1}{\Theta_{q g}\left(x, Q^{2}\right)}\left[\frac{\partial F_{2}\left(x, Q^{2}\right)}{\partial \ln Q^{2}}-\Phi_{q q}\left(x, Q^{2}\right) F_{2}\left(x, Q^{2}\right)\right] .
\end{aligned}
$$

Eq.(25) is a second-order equation which can be solved as

$$
\begin{aligned}
G\left(x, Q^{2}\right)= & \mathcal{F}\left(x, Q^{2}\right)\left[1-\frac{\mathcal{A}\left(x, Q^{2}\right)}{\Theta_{q g}\left(x, Q^{2}\right)} \mathcal{F}\left(x, Q^{2}\right)\right. \\
& +2\left(\frac{\mathcal{A}\left(x, Q^{2}\right)}{\Theta_{q g}\left(x, Q^{2}\right)} \mathcal{F}\left(x, Q^{2}\right)\right)^{2} \\
& \left.-5\left(\frac{\mathcal{A}\left(x, Q^{2}\right)}{\Theta_{q g}\left(x, Q^{2}\right)} \mathcal{F}\left(x, Q^{2}\right)\right)^{3}+\ldots .\right] \\
& =\mathcal{F}\left(x, Q^{2}\right)\left[1-\mathcal{N}+2 \mathcal{N}^{2}-5 \mathcal{N}^{3}+\ldots .\right] \\
& =\mathcal{F}\left(x, Q^{2}\right)[\mathcal{N} \mathcal{L} \mathcal{C}],
\end{aligned}
$$

where $\mathcal{N} \mathcal{L C}=\frac{\mathcal{A}\left(x, Q^{2}\right)}{\Theta_{q g}\left(x, Q^{2}\right)} \mathcal{F}\left(x, Q^{2}\right), \quad \mathcal{A}\left(x, Q^{2}\right)=$ $\frac{5}{18} \frac{27 \alpha_{s}^{2}}{160 R^{2} Q^{2}}$ and $\mathcal{F}\left(x, Q^{2}\right)=\frac{1}{\Theta_{q g}\left(x, Q^{2}\right)}\left[\frac{\partial F_{2}\left(x, Q^{2}\right)}{\partial \ln Q^{2}}-\right.$ $\left.\Phi_{q q}\left(x, Q^{2}\right) F_{2}\left(x, Q^{2}\right)\right]$. Therefore the nonlinear corrections $(\mathcal{N} \mathcal{L C} s)$ to the reduced cross section are obtained by the following forms

$\left.\sigma_{r}\left(x, Q^{2}\right)\right|_{N L C}=F_{2}\left(x, Q^{2}\right)\left\{1-\frac{y^{2}}{Y_{+}}\left(I_{L, s}\left(x, Q^{2}\right)-\frac{\Phi_{q q}\left(x, Q^{2}\right)}{\Theta_{q g}\left(x, Q^{2}\right)} I_{L, g}\left(x, Q^{2}\right)[\mathcal{N} \mathcal{L C}]\right)\right\}-\frac{\partial F_{2}\left(x, Q^{2}\right)}{\partial \ln Q^{2}}\left\{\frac{y^{2}}{Y_{+}} \frac{I_{L, g}\left(x, Q^{2}\right)}{\Theta_{q g}\left(x, Q^{2}\right)}\right\}[\mathcal{N} \mathcal{L C}]$.

\section{Higher Twist corrections:}

Introduction of higher-twist terms is one possible way to extend the DGLAP framework to low $Q^{2}$ values. Such terms have been introduced at low- $x$ values since, for the kinematics of HERA, low $Q^{2}$ is only accessed at low $x$. To better illustrate our calculations at low $Q^{2}$, we added a higher twist term in the description of the structure functions for HERA data on deep inelastic scattering at low $x$ and low $Q^{2}$ values. It can be clearly seen that our predictions with respect to the higher twist (HT) analyses are comparable with data at this region. The leading twist perturbative QCD predictions of the structure function $F_{2}$ augment by a simple higher twist term such that

$$
\begin{aligned}
F_{2}^{H T}= & F_{2}^{D G L A P}\left(1+\frac{A_{2}}{Q^{2}}\right) \\
\frac{\partial F_{2}^{H T}\left(x, Q^{2}\right)}{\partial \ln Q^{2}}= & \frac{\partial F_{2}^{D G L A P}\left(x, Q^{2}\right)}{\partial \ln Q^{2}}\left(1+\frac{A_{2}}{Q^{2}}\right) \\
& -F_{2}^{D G L A P} \frac{A_{2}}{Q^{2}} .
\end{aligned}
$$

where $A_{2}^{H T}=0.12 \pm 0.07 \mathrm{GeV}^{2}$ is a free parameter at NNLO [32-33]. Using the HT terms in Eq.(28), we can evaluate the HT corrections to the reduced cross section as 


$$
\begin{aligned}
\left.\sigma_{r}\left(x, Q^{2}\right)\right|_{H T}= & F_{2}\left(x, Q^{2}\right)\left(1+\frac{A_{2}}{Q^{2}}\right)\left\{1-\frac{y^{2}}{Y_{+}}\left(I_{L, s}\left(x, Q^{2}\right)-\frac{\Phi_{q q}\left(x, Q^{2}\right)}{\Theta_{q g}\left(x, Q^{2}\right)} I_{L, g}\left(x, Q^{2}\right)\right)\right\} \\
& -\left[\frac{\partial F_{2}\left(x, Q^{2}\right)}{\partial \ln Q^{2}}\left(1+\frac{A_{2}}{Q^{2}}\right)-F_{2}\left(x, Q^{2}\right) \frac{A_{2}}{Q^{2}}\right]\left\{\frac{y^{2}}{Y_{+}} \frac{I_{L, g}\left(x, Q^{2}\right)}{\Theta_{q g}\left(x, Q^{2}\right)}\right\} .
\end{aligned}
$$

\section{Modified Nonlinear (MNL) and Higher Twist (HT) corrections:}

To proceed further, we use the higher twist corrections to the nonlinear behavior and put them in nonlinear corrections as a modified nonlinear correction $(\mathcal{M N} \mathcal{N C})$ propose to replace the $\mathcal{N} \mathcal{L C}$ as $\mathcal{M N} \mathcal{L C} \equiv \mathcal{N} \mathcal{L C}+H T C$. The modified NLC reads

$$
\mathcal{M N} \mathcal{L C}=\frac{\mathcal{A}\left(x, Q^{2}\right)}{\Theta_{q g}^{2}\left(x, Q^{2}\right)}\left[\left\{\frac{\partial F_{2}\left(x, Q^{2}\right)}{\partial \ln Q^{2}}\left(1+\frac{A_{2}}{Q^{2}}\right)-F_{2}\left(x, Q^{2}\right) \frac{A_{2}}{Q^{2}}\right\}-\Phi_{q q}\left(x, Q^{2}\right)\left\{F_{2}\left(x, Q^{2}\right)\left(1+\frac{A_{2}}{Q^{2}}\right)\right\}\right]
$$

Following the same procedure, the modified nonlinear

can be estimated as and higher twist corrections to the reduced cross section

$$
\begin{aligned}
\left.\sigma_{r}\left(x, Q^{2}\right)\right|_{M N L C}= & F_{2}\left(x, Q^{2}\right)\left(1+\frac{A_{2}}{Q^{2}}\right)\left\{1-\frac{y^{2}}{Y_{+}}\left(I_{L, s}\left(x, Q^{2}\right)-\frac{\Phi_{q q}\left(x, Q^{2}\right)}{\Theta_{q g}\left(x, Q^{2}\right)} I_{L, g}\left(x, Q^{2}\right) \mathcal{M N} \mathcal{L C}\right)\right\} \\
& -\left[\frac{\partial F_{2}\left(x, Q^{2}\right)}{\partial \ln Q^{2}}\left(1+\frac{A_{2}}{Q^{2}}\right)-F_{2}\left(x, Q^{2}\right) \frac{A_{2}}{Q^{2}}\right]\left\{\frac{y^{2}}{Y_{+}} \frac{I_{L, g}\left(x, Q^{2}\right)}{\Theta_{q g}\left(x, Q^{2}\right)}\right\} \mathcal{M N} \mathcal{N C} .
\end{aligned}
$$

Therefore we observe that our analysis is based on the gluon and reduced cross section where the structure function and its derivatives are supposed to be known and determined from the existing experimental data.

\section{Results and Discussion}

In this section, we shall present our results that have been obtained for the gluon distribution function $G\left(x, Q^{2}\right)$ and reduced cross section $\sigma_{r}\left(x, Q^{2}\right)$ using the hard-pomeron behavior of the parton distributions. To investigate this, a general relation (i.e., Eq.(8)) between the gluon density and proton structure function and also the logarithmic slops $F_{2}$ obtained. Several methods of relating the $F_{2}$ scaling violation to the gluon density at low $x$ have been suggested previously [34-36]. Recently a similar connection between the gluon density and $\partial F_{2} / \partial \ln Q^{2}$ in FCC-eh [3] from the extension of range and precision in the measurement is suggested. Eq.(8) shows that the gluon density directly is related to $F_{2}$ and $\partial F_{2} / \partial \ln Q^{2}$. Figure 1 shows the coefficient $\frac{\Phi_{q q}}{\Theta_{q g}}$ as a function of $Q^{2}$ for the two $x$ values $1 \times 10^{-6}$ and $1 \times 10^{-3}$. The coefficient of $F_{2}$ is zero at NNLO only at $Q^{2} \simeq 200 \mathrm{GeV}^{2}$ and $Q^{2} \simeq 30 \mathrm{GeV}^{2}$ at low $\left(1 \times 10^{-6}\right)$ and moderate $\left(1 \times 10^{-3}\right) x$ values respectively. In what follows it is convenient to directly use the gluon distribution behavior with respect to $F_{2}$ and $\partial F_{2} / \partial \ln Q^{2}$. One can see that proton structure function and its derivative are supposed to be known with respect to the paremetrizations represented in Refs.[8-10]. These parameterization obtained from a combined fit of the H1 and ZEUS collaborations data [11] in a range of the kinematical variables $x<0.01$ and $0.15 \mathrm{GeV}^{2}<Q^{2}<3000 \mathrm{GeV}^{2}$.

In Fig.2 the determined gluon distribution function is 
shown in a wide range of $Q^{2}$ values for $x=1 \times 10^{-3}$ at LO upto NNLO approximation. The leading order gluon distribution is compared with the most parametrizations suggested at LO in Refs.[12-13]. The explicit forms of the gluon distributions are given in Appendix A. These results are accompanied with errors due to fit parametrizations of $F_{2}$. In Ref.[23], the effective exponents for singlet and gluon densities have been derived which they are closer to those defined by the color dipole model and hard-pomeron exponents [37]. The predictions for the gluon distribution function in the kinematic range where it has been determined by M.M.Block collaboration [8-10, 12-13] computed and compared at low values of $x$.

In Ref. [10] a new parametrization of the SF $F_{2}\left(x, Q^{2}\right)$ which describes fairly well the available experimental data on the reduced cross sections have been suggested. With respect to this new parameterization, we consider the gluon behavior in a wide range of $Q^{2}$ values at low $x$ in Fig.3. In this figure we have compared our results at LO upto NNLO approximation with those obtained by authors in Refs.[12-13] at LO approximation.

A detailed comparison has also been shown with the NNLO results from Block 2014 parameterization [10] and depicted in figure 4. In this figure our result based on the hard pomeron behavior have been presented for gluon distribution at low and moderate $Q^{2}$ values $\left(Q^{2}=3.5,5,8.5,12\right.$ and $\left.20 \mathrm{GeV}^{2}\right)$. These results have been compared with the NNLO analysis of JR09 model [38] and clearly show significant agreement over a wide range of $x$ and $Q^{2}$ values.

The nonlinear corrections (NLCs) to the gluon density are considered in a wide range of $Q^{2}$ values for different values of $x$, viz. $1 \times 10^{-3}, 1 \times 10^{-5}$ and $1 \times 10^{-7}$ respectively. In Fig.5, the effect of nonlinearity in our results is investigated for $R=2 \mathrm{GeV}^{-1}$ at NNLO approximation. One can see that obtained nonlinear corrections for gluon distribution function are larger for very low $x$ values $\left(x<10^{-3}\right)$ at low $Q^{2}$ values $\left(1<Q^{2}<10 \mathrm{GeV}^{2}\right)$ then another domains. Fig.6 represent linear and nonlinear results of of gluon distribution function for $R=2 \mathrm{GeV}^{-1}$ at NNLO that have been computed from Eqs.(8) and (26) for $Q^{2}=3.5,8.5$ and $20 \mathrm{GeV}^{2}$ respectively. It would appear that the effect of nonlinearity at low $x$ should observe for low and moderate $Q^{2}$. The nonlinear corrections can be neglected at large values of $Q^{2}$, so we expect that our results are consistent in the kinematic region $x \leq 0.01$ and moderate $Q^{2}$ with other results. From these figurs, it is observed that the NNLO nonlinear corrections (NNLO+NLCs) show tamed behavior compared with only NNLO approximation.

In Fig.7 we display our NNLO results for $\sigma_{r}$ with respect to the parameterization of $F_{2}$ [10] at different proton beam energies $E_{p}(=920,820$ and $575 \mathrm{GeV})$, relevant for most H1 measurements [39-40], where the turnover at small $x$ becomes more pronounced at smaller energies because of the larger values of $y$. The data are taken with positrons of energy $E_{e}=27.6 \mathrm{GeV}$ corresponding to the center of mass energies $\sqrt{s}=2 \sqrt{E_{e} E_{p}}[\mathrm{GeV}]$. In Fig.8 the NNLO predictions at $\sqrt{s}=300.9 \mathrm{GeV}$ are compared with H1 data [39] as accompanied with total errors. These small- $x$ predictions are fully compatible with the H1 data presented in [39]. The modified nonlinear and higher twist corrections are depicted and compared with the linear dependence in these figures. We have also performed an analysis to check the sensitivity of the modified nonlinear and higher twist corrections in reduced cross section results.

We have calculated the $Q^{2}$-dependence, at low $x$, of the reduced cross section $\sigma_{r}\left(x, Q^{2}\right)$ (i.e., Eq.(30)) in the NNLO approximation. Results of calculations and comparison with data of the H1-Collaboration [39] are presented in Fig.10, where the solid line correspond to the extracted $\sigma_{r}$ in the NNLO approximations. Calculations have been performed at fixed value of the inelasticity $y, y=0.675$, allowing the Bjorken variable $x$ to vary in the interval $\left(3 \times 10^{-5}<x<7 \times 10^{-4}\right)$ when $Q^{2}$ varies in the interval $\left(1.5<Q^{2}<40 \mathrm{GeV}^{2}\right)$. To illustrate better the modified nonlinear and higher twist corrections at low $Q^{2}$ values, we have plotted MNL+HT predictions to the NNLO results in Fig.10. It may lead to the better determinations of the reduced cross section specially at low $Q^{2}$.

\section{Summary and Conclusion}

We presented Eqs.(8-10) for the extraction of the gluon distribution function $G\left(x, Q^{2}\right)$ at low $x$ in the leading-order upto next-to-next-to-leading order from the $F_{2}\left(x, Q^{2}\right)$ and its $\ln Q^{2}$ derivative. Then rendered the results for the reduced cross section $\sigma_{r}\left(x, Q^{2}\right)$ in a wide range of $Q^{2}$ values. The reduced cross section behavior is considered at low $x$ with respect to the $F_{2}$ parameterization. These results based on an effective exponent are in good agreement with the experimental data at low $x$ values. We have studied the effects of adding the nonlinear GLR-MQ corrections to the NNLO linear behavior of the gluon distribution function and reduced cross section. These results are close to the data for low- $Q^{2}$ values as we have discussed the meaning of these findings from the points of view of modified nonlinear and higher twist corrections added to the structure function.

\section{Acknowledgments}

The authors appreciate the Research Council of Razi University for official support of this work. G.R.Boroun 
thanks the Department of Physics of the CERN-TH for their warm hospitality. Also authors would like to thank H.Khanpour for discussions which completed this study.

\section{Appendix A}

The gluon distribution is derived from the leading order DGLAP evolution equation for $F_{2}\left(x, Q^{2}\right)$ in a domain $x \leq 0.09$ and $0.11 \mathrm{GeV}^{2} \leq Q^{2} \leq 1200 \mathrm{GeV}^{2}$. Solution for $G\left(x, Q^{2}\right)$ using a Froissart bounded structure function $F_{2}\left(x, Q^{2}\right)$ is obtained in terms of a quadratic polynomial in $\ln (1 / x)$ with quadratic polynomial coefficients in $\ln \left(Q^{2}\right)$ as $[12]$

$$
\begin{gathered}
G\left(x, Q^{2}\right)=-0.459-0.143 \ln \left(Q^{2}\right)-0.0155 \ln ^{2}\left(Q^{2}\right) \\
+\left[0.231+0.00971 \ln \left(Q^{2}\right)-0.0147 \ln ^{2}\left(Q^{2}\right)\right] \ln (1 / x) \\
+\left[0.0836+0.06328 \ln \left(Q^{2}\right)+0.0112 \ln ^{2}\left(Q^{2}\right)\right] \ln ^{2}(1 / x) .(31
\end{gathered}
$$

The above method generalized to the case of massive quarks directly from $F_{2}\left(x, Q^{2}\right)$ by Laplace transforms to the DGLAP equation [13]. The gluon distribution function for $n_{f}=4$ at $Q^{2}=M_{b}^{2}$ with $M_{b}=4.5 \mathrm{GeV}$ is obtained with an expression quadratic in both $\ln (1 / x)$ and $\ln \left(Q^{2}\right)$ by the following form

$$
G_{4}\left(x, Q^{2}\right)=\frac{3}{5} \mathcal{H}_{3}\left(x, Q^{2}\right)=\mathcal{H}_{4}\left(x, Q^{2}\right),
$$

where

$$
\begin{aligned}
& \mathcal{H}_{3}\left(x, Q^{2}\right)=-2.94-0.359 \ln \left(Q^{2}\right)-0.101 \ln ^{2}\left(Q^{2}\right) \\
+ & {\left[0.594-0.0792 \ln \left(Q^{2}\right)-0.000578 \ln ^{2}\left(Q^{2}\right)\right] \ln (1 / x) } \\
& +\left[0.168+0.138 \ln \left(Q^{2}\right)+0.0169 \ln ^{2}\left(Q^{2}\right)\right] \ln ^{2}(1 / x) .(33)
\end{aligned}
$$

\section{REFERENCES}

1. M. Klein, Ann. Phys.528, 138 (2016).

2. R.A.Khalek et al., arXiv:1906.10127 [2019].

3. A. Abada et al., [FCC Collaborations], Eur.Phys.J.C79, 474(2019).

4. A.Donnachie and P.V.Landshoff, Phys.Lett.B 470, 273(1999).

5. M.Froissart, Phys.Rev.123, 1053 (1961).

6. Yu.L.Dokshitzer, Sov.Phys.JETP 46, 641(1977); G.Altarelli and G.Parisi, Nucl.Phys.B 126, 298(1977); V.N.Gribov and L.N.Lipatov, Sov.J.Nucl.Phys. 15, 438(1972).

7. H.Khanpour et al., Phys.Rev.C95, 035201(2017); H.Khanpour, Phys.Rev. D99, 054007(2019).

8. E. L. Berger, M. M. Block and C. I. Tan, Phys. Rev. Lett. 98, 242001 (2007); M. M. Block, E. L. Berger and C. I. Tan, Phys. Rev. Lett.97, 252003 (2006).

9. M. M. Block, L. Durand, P. Ha and D. W. McKay, Phys. Rev.D 84, 094010 (2011); Phys. Rev.D 88, no. 1, 014006 (2013).

10. M. M. Block, L. Durand and P. Ha, Phys. Rev.D 89, no. 9, 094027 (2014).

11. F. D. Aaron et al. [H1 and ZEUS Collaborations], JHEP1001, 109 (2010).

12. M. M. Block, L. Durand and D. W. McKay, Phys. Rev.D 77, 094003 (2008); Phys. Rev.D 79, 014031 (2009).

13. M.M.Block, Eur.Phys.J.C65, 1(2010); M. M. Block and L. Durand, arXiv:0902.0372 hep-ph](2009).

14. K.Prytz, Phys.Lett.B311,286(1993); A.V.Kotikov and G.Parente, Phys.Lett.B379, 195(1996); G.R.Boroun and B.Rezaei, Chin.Phys.Lett.23, 324(2006).

15. G.R.Boroun and B.Rezaei, Eur.Phys.J.C73, 2412(2013); S.Zarrin and G.R.Boroun, Nucl.Phys.B922, 126 (2017); M.Lalung et al., Int.J.Theor.Phys.56, 3625(2017); M.Devee, arXiv:1808.00899(2018).

16. A.V.Kotikov, Phys.Atom.Nucl,80, 572 (2017).

17. N.Yu.Chernikova and A.V.Kotikov, JETP Lett.105, $223(2017)$.

18. A.Donnachie and P.V.Landshoff, Phys.Lett.B 550, 160(2002).

19. W.L. van Neerven, A.Vogt, Phys.Lett.B 490, 111(2000).

20. A.Vogt, S.Moch, J.A.M.Vermaseren, Nucl.Phys.B 691, 129(2004).

21. B.G. Shaikhatdenov, A.V. Kotikov, V.G. Krivokhizhin, G. Parente, Phys. Rev. D 81, 034008(2010).

22. K Golec-Biernat and A.M.Stasto, Phys.Rev.D 80, 014006(2009); R.D.Ball et al., Eur.Phys.J. C76, $383(2016)$.

23. B.Rezaei and G.R.Boroun, Eur.Phys.J.A55, 66(2019); G.R.Boroun, Eur.Phys.J.A50, 69(2014).

24. L.A.Anchordoqui et al., arXiv:1902.10134 hep-ph].

25. G.Altarelli and G.Martinelli, Phys.Lett.B76, 
$89(1978)$

26. S.Moch, J.A.M.Vermaseren and A.Vogt, Phys.Lett.B606, 123(2005).

27. A. H. Mueller and J. Qiu, Nucl. Phys. B268(1986)427; L. V. Gribov, E. M. Levin and M. G. Ryskin, Phys. Rep.100, (1983)1.

28. G. R. Boroun and B. Rezaei, Chin. Phys. Lett.32, (2015) no.11, 111101; B. Rezaei and G. R. Boroun, Phys. Lett. B692 (2010) 247; G. R. Boroun, Eur. Phys. J. A43 (2010) 335.

29. N. N. Nikolaev and W. Schäfer, Phys. Rev. D74(2006)014023.

30. K. J. Eskola et al., Nucl. Phys. B660(2003)211.

31. R. Fiore, P. V. Sasorov and V. R. Zoller, JETP Letters 96(2013)687; R. Fiore, N. N. Nikolaev and V. R. Zoller, JETP Letters 99(2014)363.

32. A.M.Cooper-Sarkar, arXiv:1605.08577v1 [hep-ph] 27 May 2016; I.Abt et.al., arXiv:1604.02299v2 [hep-ph] 11 Oct 2016.
33. F.D. Aaron et al. [H1 Collaboration], Eur.Phys.J. C63, 625(2009).

34. K.Prytz, Phys.Lett.B311, 286(1993); Phys.Lett.B332, 393(1994).

35. M.B.Gay Ducati and P.B.Goncalves, Phys.Lett.B390, 401(1997).

36. G.R.Boroun, Journal of Experimental and Theoretical Physics, 111, 566(2010); N.N.Nikolaev and B.G.Zakharov, Phys.Lett. B332, 184(1994).

37. N.N.Nikolaev and B.G.Zakharov, Phys.Lett.B332, 184(1994); N.N.Nikolaev and V.R.Zoller, Phys.Atom.Nucl.73, 672 (2010).

38. P.Jimenez-Delgado and E.Reya, Phys.Rev.D79, 074023(2009).

39. C.Adloff et al., [H1 Collaboration], Eur.Phys.J.C.21, $33(2001)$.

40. F.D. Aaron et al., [H1 Collaboration], Phys.Lett.B.665, 139(2008). 


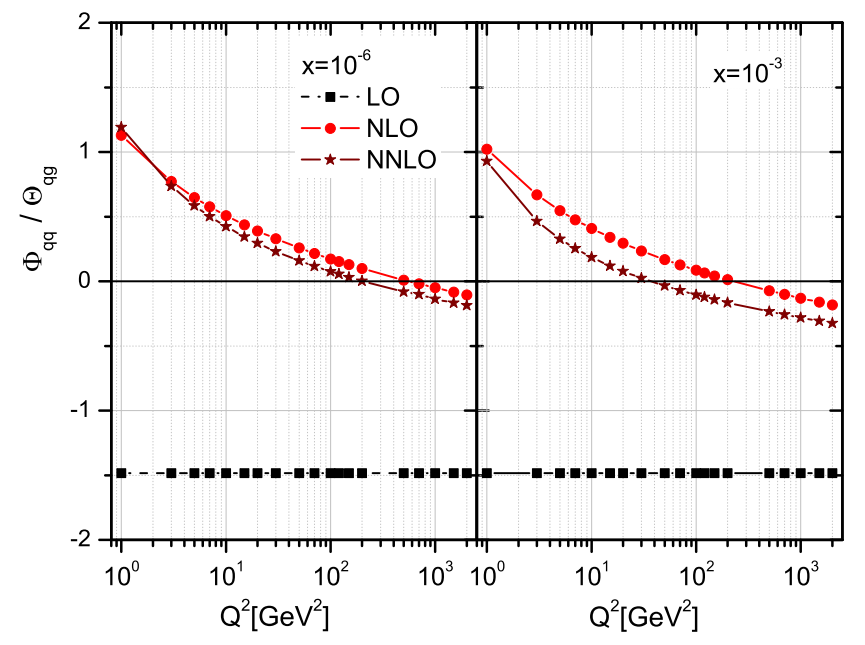

FIG. 1: Plot of the ratio $\frac{\Phi_{q q}}{\Theta_{q g}}$ as a function of $Q^{2}$ for two $x$ $\left(1 \times 10^{-6}\right.$ and $\left.1 \times 10^{-3}\right)$.

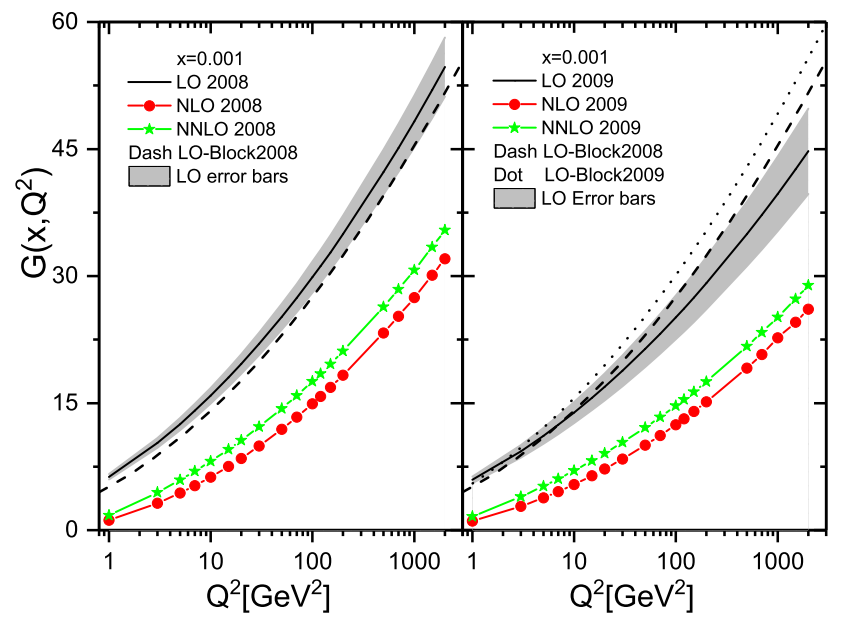

FIG. 2: The leading-order up to the next-to next-to leading order gluon distributions in comparison with the leading-order results of Block model [12-13]. The error bars are due to the $F_{2}$ parameterization at $\mathrm{LO}$ approximation. 


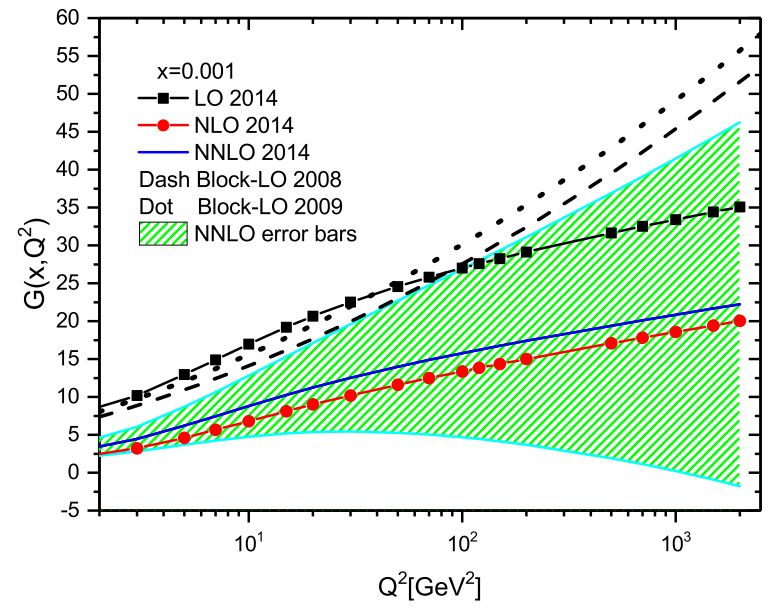

FIG. 3: The leading-order up to the next-to next-to leading order gluon distributions from the new $F_{2}$ parameterization [10] compared with the leading-order results of Block model [12-13]. The error bars are due to the $F_{2}$ parameterization at NNLO approximation. 

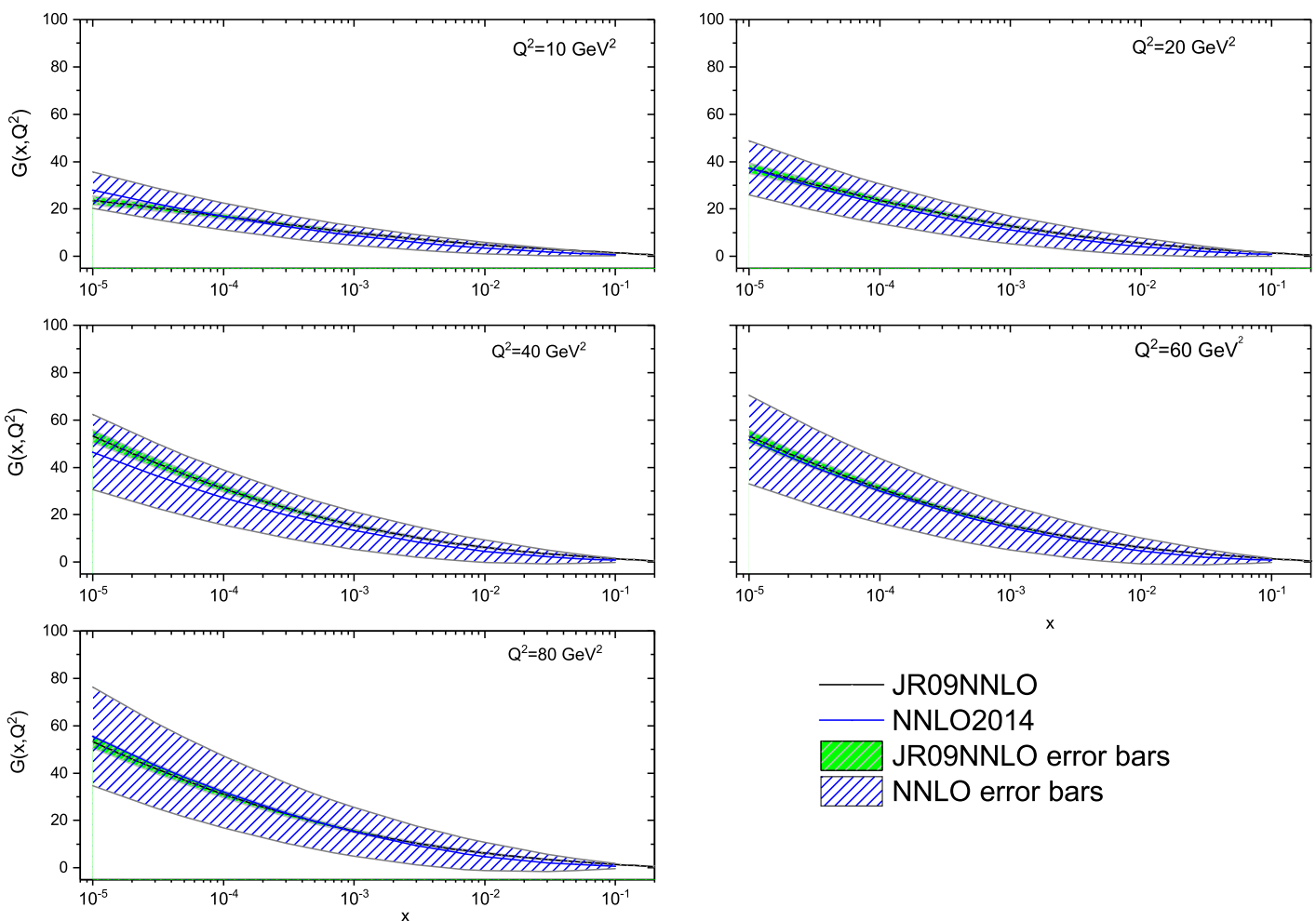

JR09NNLO

NNLO2014

JR09NNLO error bars

VIIIA NNLO error bars

FIG. 4: The NNLO gluon distributions from the new $F_{2}$ parameterization [10] and its comparison with the results from JR09NNLO global QCD analysis [38] at $Q^{2}=10,20,40,60$ and $80 \mathrm{GeV}^{2}$. The error bars are due to the $F_{2}$ parameterization at NNLO approximation and JR09 NNLO analysis. 


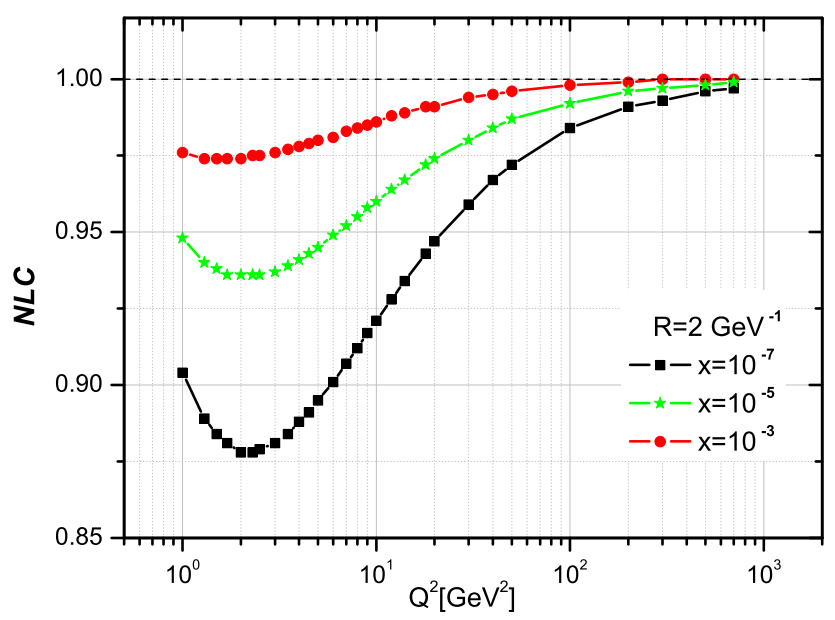

FIG. 5: Nonlinear correction (NLC) effects for $R=2 \mathrm{GeV}^{-1}$ in a wide range of $Q^{2}$ at low and very low $x$ values.

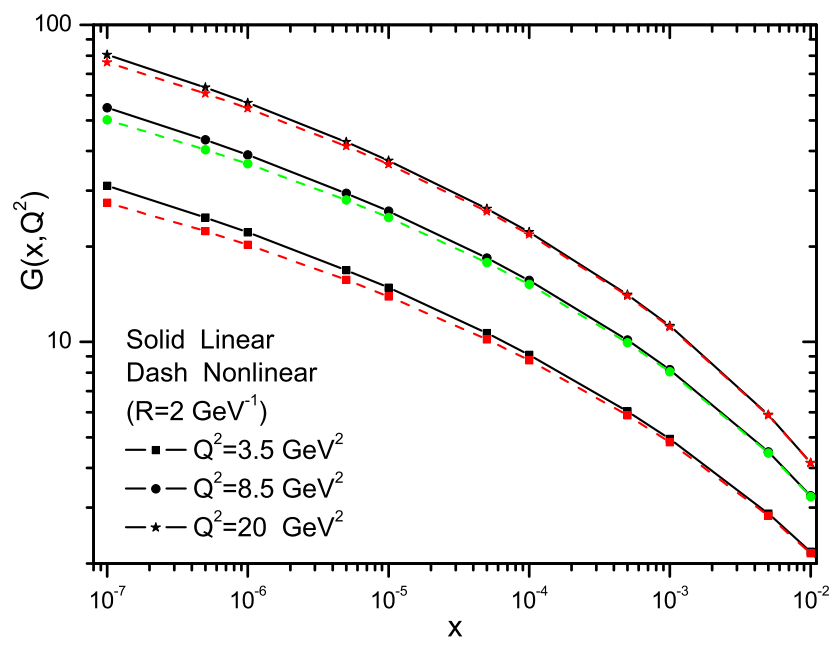

FIG. 6: x-evolution of linear and nonlinear gluon distribution function at NNLO approximation for three fixed $Q^{2}$. 


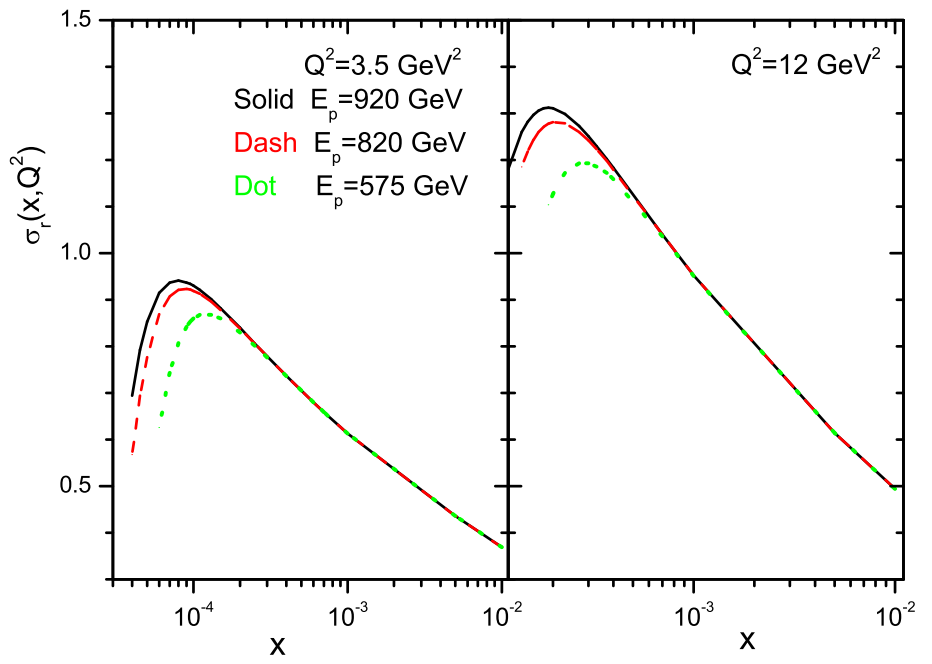

FIG. 7: The NNLO predictions for $\sigma_{r}\left(x, Q^{2}\right)$ but for different proton beam energies $E_{p}$ at $Q^{2}=3.5$ and $12 \mathrm{GeV}^{2}$. Notice that the curves terminate when $y=1$. 


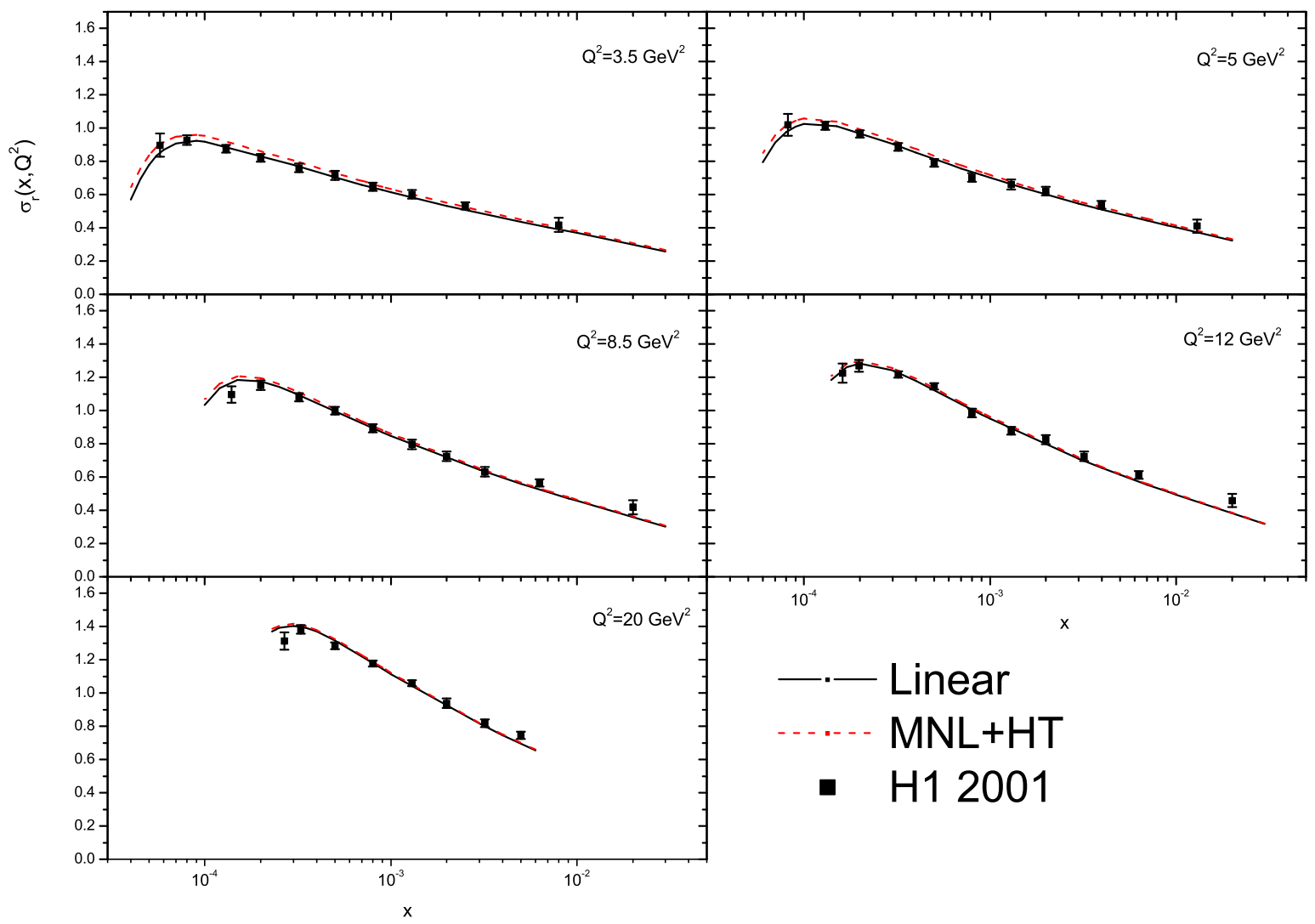

FIG. 8: The NNLO predictions for the reduced DIS cross section. The modified nonlinear (MNL) and higher twist (HT) corrections represent our results at NNLO. The $\mathrm{H} 1$ data for some representative fixed values of $Q^{2}$ are taken from [39]. 


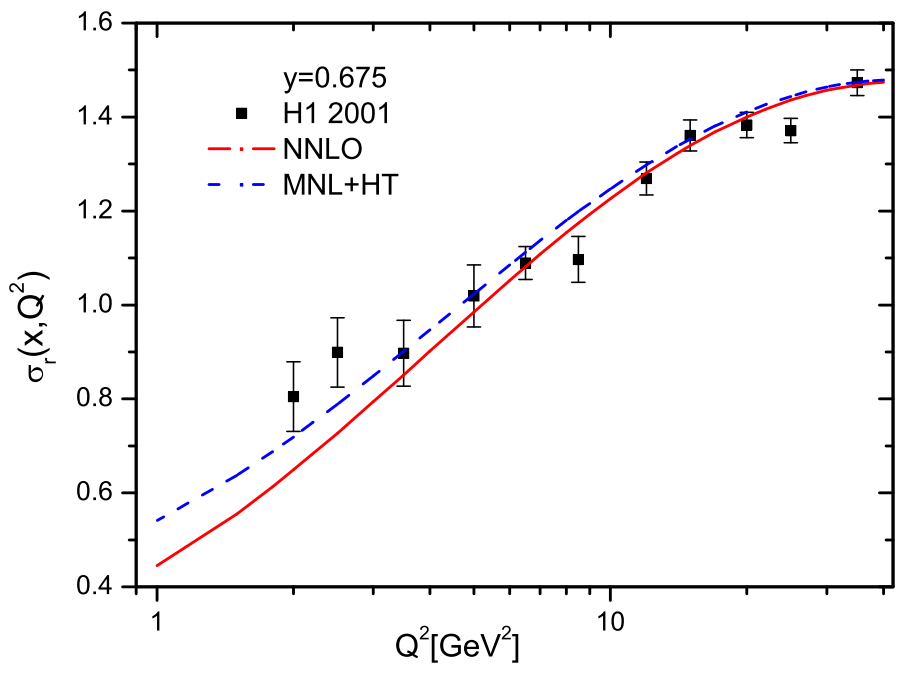

FIG. 9: The NNLO predictions for the reduced DIS cross section at high inelasticity available. The $\mathrm{H} 1$ data for some representative fixed values of $Q^{2}$ are taken from [39] as accompanied with total errors. Modified nonlinear and higher twist corrections compared with linear results in a wide range of $Q^{2}$. 